\title{
Coinfection of Bartonella spp. and Borrelia Burgdorferi in Ixodes Scapularis Using PCR Assay, a Case Study in Nova Scotia
}

\author{
James Kho ${ }^{1}$, Chelsea Colbourne ${ }^{1}$, Emma Bent ${ }^{1}$, Amal El Nabbout ${ }^{1}, \&$ Tatiana Rossolimo ${ }^{1}$ \\ ${ }^{1}$ Department of Biology, Dalhousie University, Halifax, Canada \\ Correspondence: James Kho, Department of Biology, Dalhousie University, 1355 Oxford St., Halifax, NS B3H 4R2, \\ Canada
}

Received: October 13, 2021

Accepted: November 15, $2021 \quad$ Online Published: November 25, 2021

doi:10.5539/ijb.v13n2p57

URL: https://doi.org/10.5539/ijb.v13n2p57

\begin{abstract}
Coinfection of vector species can provide more insight into the complex relationship between zoonotic pathogens and its host. Ixodes scapularis (Say) or the deer-tick in particular is an important species in North America because of its exceptional ability as a vector that can transmit zoonotic diseases such as Lyme and Cat Scratch Disease (CSD). In recent years, many studies have suggested a possible link between the coinfection of Borrelia burgdorferi, the causative agent of Lyme, with other tick-borne bacteria such as Bartonella spp., the causative agent of CSD, as partly responsible for the symptoms associated with Chronic Lyme Disease or Post-Treatment Lyme Disease Syndrome. This study investigates the prevalence of Bartonella spp. and Borrelia burgdorferi in Ixodes scapularis using Polymerase Chain Reaction (PCR) assay to potentially find a link between the two of the most common tick-borne pathogens found in Nova Scotia. Standard PCR using primers targeted at the two bacterial species were conducted on 157 I. scapularis ticks collected in Nova Scotia. Overall, we found high prevalence for both bacteria at $75.16 \%$ for Bartonella spp. and $47.13 \%$ for $B$. burgdorferi with no significant differences between the sex of the ticks. Interestingly, all the ticks positive for $B$. burgdorferi were also positive for Bartonella spp. which implies that the coinfection rate between $B$. burgdorferi and Bartonella spp. is $47.13 \%$. We report one of the highest coinfection rates for B. burgdorferi and Bartonella spp. in I. scapularis, consistent with the current trends of increasing tick presence in North America.
\end{abstract}

Keywords: Bartonella spp., Borrelia burgdorferi, coinfection, Ixodes scapularis, PCR, ticks

\section{Introduction}

\subsection{Coinfection in Ticks}

Coinfection in arthropod vectors is an ongoing topic of research. Studies range from investigating the effect of coinfection on transmission to and from the host (Levin and Fish, 2000; Steiner et al., 2014; Diuk-Wasser et al., 2016) to the clinical effects of coinfection (Eskow et al., 2001; Diuk-Wasser et al., 2016). One of the most well studied group of arthropod vectors with regards to coinfection is the ticks. Hard ticks (F. Ixodidae) in particular is of interest due to their clinical significance. In Eastern Canada, Ixodes scapularis (Say) is the species that is responsible for the majority of tick-borne diseases including Lyme disease (Curry et al., 2017; Lloyd and Hawkins, 2018; Carey et al., 2019; Foley-Eby et al., 2020).

\subsection{Ixodes Scapularis}

Ixodes scapularis, more commonly known as either the deer-tick or the black-legged tick, is found throughout eastern North America. They are common vectors for Borrelia spp., Bartonella spp. of bacteria, as well as an assortment of other infectious pathogens (Corona \& Schwartz, 2015; Karasartova et al., 2018). One of the reasons that I. scapularis is able to accommodate a variety of pathogens is in part due to their life cycle and the uniquen requirements for each individual life stage. Once the egg hatches, each life stage (larva, nymph and adult) corresponds to distinct hosts (Edwards and Rawlings, 2012), including a variety of wild and domestic fauna. Larvae and nymphs tend to feed on birds or small rodents. Adults typically feed on larger fauna, for example, white-tailed deer, domestic dogs, and humans (Kim et al., 2016). By feeding on different hosts, the probability of the tick being infected by and/or transmitting pathogens increases (Parola and Raoult, 2001; Sonenshine, 1991). Among all the bacteria that can be transmitted, Borrelia burgdorferi sensu lato and Bartonella spp. are among the most documented bacteria species transmitted in Eastern Canada (Lloyd and Hawkins, 2018; Carey et al., 2019; Foley-Eby et al., 2020). 


\subsection{Borrelia Burgdorferi}

Borrelia burgdorferi is the causative agent for one of the most concerning tick-borne diseases, Lyme disease (Ginsberg et al., 2017). B. burgdorferi is a spirochete bacterium that enters the host through the blood meal of a tick. The bacterium can then infect several organs throughout the body through the bloodstream and cause Lyme borreliosis or Lyme disease (Ruef, 2011; Lloyd and Hawkins, 2018). Recent studies have suggested a link between symptoms of Lyme disease with coinfection of other bacteria that inhabit the tick vector including Bartonella spp. (Holden et al., 2006; Sytykiewicz et al., 2012).

\subsection{Bartonella Spp.}

Bartonella spp. are gram negative, aerobic, facultative intracellular bacteria (Müller et al., 2016). Bartonella spp. can cause various clinical symptoms within humans depending on the species or sub-species (Chang et al., 2001). While there is no evidence suggesting human transmission of Bartonella spp. from ticks there is the possibility of co-infection with Borrelia burgdorferi as previously found in mice (Hofmeister et al., 1998; Müller et al., 2016). Understanding the infection prevalence within ticks for these bacteria species is essential for better assessment of the exposure risk.

\subsection{Aim of Study}

This study aims to identify the coinfection rate of Borrelia burgdorferi, and Bartonella spp. in I. scapularis found in Nova Scotia, Canada. The number and variety of bacterial species found in each tick will be established via DNA isolation and PCR. This report will provide the foundation needed to investigate the relationships between bacterial co-infections and its host in an important local vector.

\section{Methods}

\subsection{Sample Collection}

Ixodes scapularis ticks were collected in the wild from various areas in Nova Scotia and tested for Bartonella spp. and Borrelia burgdorferi using Polymerase Chain Reaction (PCR) assay. The detailed protocols followed for sample collection, DNA extraction, PCR for Bartonella spp., and gel electrophoresis can be found in Carey et al. (2019).

Table 1. A list of the specific primers used in this study to detect Bartonella spp., and Borrelia burgdorferi

\begin{tabular}{|c|c|c|c|c|c|}
\hline \multicolumn{6}{|c|}{ Bartonella spp. } \\
\hline Primer name & Gene & Sequence (5'-3') & Amplicon Size & Annealing Temperature & Source \\
\hline P-24E & 16S-rRNA & $\begin{array}{l}\text { GGAATTCCCTCCTTCAGTTAGGCTG } \\
\text { G }\end{array}$ & 279 bp & $56^{\circ} \mathrm{C}$ & $\begin{array}{l}\text { Eskow et al. } \\
\text { (2001); }\end{array}$ \\
\hline $\mathrm{P}-12 \mathrm{~B}$ & 16S-rRNA & $\begin{array}{l}\text { CGGGATCCCGAGATGGCTTTTGGA } \\
\text { GATTA }\end{array}$ & & & Carey et al. (2019) \\
\hline \multicolumn{6}{|c|}{ Borrelia burgdorferi } \\
\hline Primer name & Gene & Sequence (5'-3') & Amplicon Size & Annealing Temperature & Source \\
\hline OspA_Out_R1 & $O s p A$ & GTTAGCAGCCTTGAC GAGA & $272 \mathrm{bp}$ & $55^{\circ} \mathrm{C}$ & Ogden et al. \\
\hline OspA_Out_F1 & OspA & GATACTAGTGTTTTGCCATC & & & (2006); \\
\hline OspA_In_R1 & OspA & GCGTTTCAGTAGATTTGCCTG & $214 \mathrm{bp}$ & $58^{\circ} \mathrm{C}$ & Patterson et al. \\
\hline OspA_In_F1 & OspA & TCAAGTGTGGTTTGACCTAG & & & (2017) \\
\hline FlaB_Out_R1 & FlaB & $\begin{array}{l}\text { AATTGCATACTCAGTACTATTCTTT } \\
\text { ATAGAT }\end{array}$ & $612 \mathrm{bp}$ & $55^{\circ} \mathrm{C}$ & \\
\hline FlaB_Out_F1 & FlaB & $\begin{array}{l}\text { AAGTAGAAAAAGTCT } \\
\text { TAGTAAGAATGAAGGA }\end{array}$ & & & \\
\hline FlaB_In_R1 & FlaB & $\begin{array}{l}\text { GAAGGTGCTGTAGCAGGTGCTGGC } \\
\text { TGT }\end{array}$ & $390 \mathrm{bp}$ & $58^{\circ} \mathrm{C}$ & \\
\hline FlaB_In_F1 & FlaB & $\begin{array}{l}\text { CACATATTCAGATGCA } \\
\text { GACAGAGGTTCTA }\end{array}$ & & & \\
\hline
\end{tabular}

\subsection{Testing for Bartonella spp. Prevalence}

Briefly, DNA from half ticks was extracted using AquaGenomic solution (Multitarget Pharmaceuticals, Colorado, USA) as per the developer's instructions. The extracted DNA samples were then stored in a $-18^{\circ} \mathrm{C}$ freezer until PCR (Carey et al., 2019). PCR was done using GoTaq Green (Promega, Maddison, USA) master mix. The primers P-24E and P-12B were used to amplify a region of the $16 S$ rRNA gene-specific to Bartonella spp. from each tick sample (Table 1). Agarose gel (2.0\%) and SYBR Safe Green were used to visualize the amplicon. 


\subsection{Testing for Borrelia Burgdorferi Prevalence}

In addition to testing for Bartonella spp., the same samples were tested for the prevalence of Borrelia burgdorferi using nested PCR procedure and primers outlined in Patterson et al. (2017) and later updated in Wills et al. (2018). Nested PCR is a modification of the standard PCR procedure to target smaller amplicons better using two rounds of PCR reactions, Primary and Nested (Massung et al., 1998). The Primary reaction will generate an amplicon from the DNA sample, while the Nested reaction will then generate a more specific amplicon using the Primary reaction's amplicon (Massung et al., 1998; Wills et al., 2018). All PCR amplifications were done on an Eppendorf Mastercycler ep Gradient S.

Two genes were used to assess the prevalence of Borrelia burgdorferi: FlagellinB (FlaB) and Outer surface protein A $(O s p A)$, the former corresponding to the flagellum's primary filament protein while the latter is a lipoprotein that is involved in tick midgut colonization (Patterson et al., 2017; Wills et al., 2018). All the primers were obtained from Sigma-Aldrich and are outlined in Table 1.

After PCR, all amplicons were visualized on a $2.0 \%$ agarose gel $(2 \mathrm{~g}$ agarose, $100 \mathrm{~mL}$ of $0.5 \mathrm{x}$ Tris-Borate-EDTA (TBE) buffer, and $5 \mu \mathrm{L}$ SYBR Safe Green). $5 \mu \mathrm{L}$ of samples and $5 \mu \mathrm{L}$ of 100 bp DNA Ladder (GeneDireX) were then loaded into the gel which ran for 1 hour at 100V. Each gel was then photographed for further analysis using a Canon EOS Rebel T5.

\subsection{Assessing Co-infection and General Precaution}

Co-infection was assessed based on the prevalence of both Bartonella spp. and Borrelia burgdorferi in a sample. Percent prevalence values were calculated for each as well as both bacteria (number of positive samples/number of tested samples $\mathrm{x} 100$ ).

In the case of $B$. burgdorferi, if a positive amplicon for $O s p A$ is detected but not $F l a B$, we will consider the sample negative due to $O s p A$ being prone to false-positives; however if a positive amplicon for FlaB is detected but not $O s p A$, we will consider the sample positive due to the chance that there may be related Borrelia spp. in the sample (more information in Wills et al., 2018).

A Chi-square goodness of fit test was done to determine a correlation between tick sex and the infection prevalence of both bacteria. To determine whether there is variance around the means for infection prevalence, a one-way ANOVA was done. In addition, to investigate the relationship between the two bacteria's infection prevalence, a linear regression analysis with confidence level of 95\% was conducted. All statistical analyses were done in Microsoft Excel v2005 and R v.4.0.1.

All experimental reactions and solutions were prepared in a certified Biological Safety Cabinet. UV and 95\% ethanol were used as sterilizing agents during sample preparation, DNA extraction, and PCR prep.

\section{Result}

\subsection{Infection Prevalence and Sex of Ixodes Scapularis}

A total of 157 Ixodes scapularis ticks ( 75 male and 82 female) were tested in this study. All 157 samples were tested using PCR for the presence of Borrelia burgdorferi and Bartonella spp.. Overall, Bartonella spp. has higher infection prevalence than B. burgdorferi in both sexes. Table 2 shows the results for the B. burgdorferi and Bartonella spp. PCR tests separated by sex.

Table 2. Table showing the number of Ixodes scapularis ticks tested using PCR of OspA and FlaB targeted primers for Borrelia burgdorferi, and $16 \mathrm{~S}$ rRNA targeted primers for Bartonella spp. separated by sexes

\begin{tabular}{lccc}
\hline Borrelia burgdorferi & & Negative & Total \\
\hline Male & Positive & 37 & 75 \\
Female & 38 & 46 & 82 \\
Total & 36 & 83 & 157 \\
\hline Bartonella spp. & 74 & Negative & Total \\
& & 20 & 75 \\
Male & Positive & 19 & 82 \\
Female & 55 & 39 & 157 \\
Total & 63 & $5 p . i s$ & \\
\hline
\end{tabular}

Table 2 suggests that number of ticks that were positive with Bartonella spp. is around two thirds of the overall sample size. This pattern appears to be consistent regardless of the sex of the ticks. For B. burgdorferi, the positive samples were almost on par with the negative samples for the male I. scapularis samples but there is slightly less infection 
prevalence in the female ticks. Looking at the total proportion of samples tested, there is almost triple the amount of positive Bartonella spp. samples compared to the negative samples, and there are slightly more negative samples for Borrelia burgdorferi compared to positive samples.

A visual representation of the ratio of the two bacteria infection (in percent) separated by sex is shown in Figure 1 below.

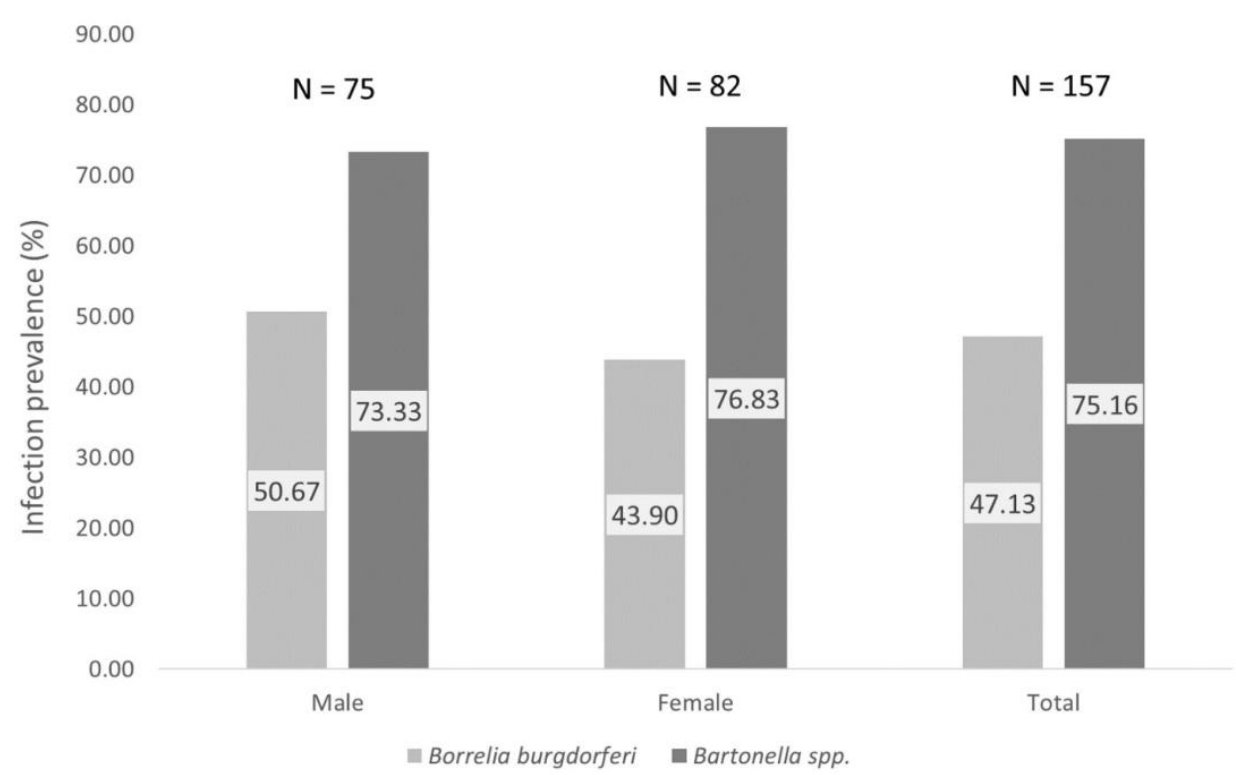

Figure 1. Bar graph showing the percent infection prevalence of Borrelia burgdorferi and Bartonella spp. in Ixodes scapularis samples separated into male $(\mathrm{N}=75)$, female $(\mathrm{N}=82)$, and total combined $(\mathrm{N}=157)$. The light grey bars represent positive PCR products from the primers targeting $O s p A$ and FlaB genes for B. burgdorferi, while the darker bars indicate positive Bartonella spp. PCR results from primers targeting $16 S r R N A$ gene

Overall, the infection prevalence of B. burgdorferi is less than Bartonella spp., which is consistent in both male and female ticks (Figure 1). A Chi-square goodness of fit test on the each of the bacteria data from Table 2 gave a $P$ values of 0.719 for Borrelia burgdorferi and 0.068 for Bartonella spp. which suggests that the sex of the tick is independent to either bacteria's infection prevalence.

\subsection{Coinfection of B. Burgdorferi and Bartonella Spp.}

Looking at the raw data alone, one interesting observation is that all the Ixodes scapularis ticks that were infected with Borrelia burgdorferi were also infected with Bartonella spp. (Data not shown). Figure 1 suggests a high coinfection rate of $47.13 \%(\mathrm{~N}=157)$ between Bartonella spp. and B. burgdorferi in Ixodes scapularis ticks. Two statistical analyses were conducted to further explore the relationship between B. burgdorferi and Bartonella spp. infection in these tick samples (Table 3).

Table 3. (A.) A one-way ANOVA comparing the infection prevalence of Borrelia burgdorferi and Bartonella spp. in Ixodes scapularis ticks $(\mathrm{N}=157)$. The P-value of 2.17E-07 suggest a significant difference between the two means. (B.) Part of the results for a linear regression analysis using B. burgdorferi as the dependent variable. The positive Coefficients value as well as a $\mathrm{P}$ value of $2.07 \mathrm{E}-13$ suggests a statistically significant positive linear relationship between the two infection prevalence

A.

\begin{tabular}{lllllll}
\hline Source of Variation & $S S$ & $d f$ & $M S$ & $F$ & $P$-value & $F$ crit \\
\hline Between Groups & 6.16 & 1 & 6.16 & 28.1 & $2.17 \mathrm{E}-07$ & 3.87 \\
Within Groups & 68.4 & 312 & 0.219 & & & \\
Total & 74.6 & 313 & & & & \\
\hline
\end{tabular}


B.

\begin{tabular}{lcccc}
\hline Linear Regression & Coefficients & Standard Error & t Stat & $P$-value \\
\hline Intercept & $1.66 \mathrm{e}-15$ & 0.067 & 2.46 & 1 \\
B. burgdorferi from Bartonella spp. & 0.627 & 0.078 & 8.05 & $2.07 \mathrm{E}-13$
\end{tabular}

A One-way ANOVA suggests a significant difference in infection prevalence between Borrelia burgdorferi and Bartonella spp. at a $\mathrm{P}$ value of $2.17 \mathrm{e}-7$, and a linear regression analysis suggests a positive relationship for $B$. burgdorferi with Bartonella spp. (Table 3). Altogether, these statistical analyses suggest a potential correlation of coinfection between B. burgdorferi and Bartonella spp. and that the infection prevalence data between the two bacteria are statistically significant.

\section{Discussion}

\subsection{General Findings and role of Sex in Prevalence}

Sex has been suggested as one of the variables that can influence the physiology of ticks (Meeüs et al., 2002; Wójcik-Fatla et al., 2006; El Nabbout et al., 2018; Batool et al., 2021). In this study, we tested 157 Ixodes scapularis ticks for Borrelia burgdorferi and Bartonella spp. using conventional PCR and gel electrophoresis techniques to determine coinfection between the two bacteria species and between the sexes of the ticks. When looking at $B$. burgdorferi infection between sexes, there were no significant differences between the male $(50.67 \%)$ and female (43.9\%) I. scapularis ticks (Chi-square goodness of fit test, P-value: 0.40). The same trend was observed for Bartonella spp. between male (73.33\%) and female (76.83) I. scapularis ticks (Chi-square goodness of fit test, P-value 0.07). These results suggest that infection of these bacteria is consistent between sexes and is particularly important for the consideration of male ticks in further studies. Male ticks are often overlooked in studies due to their less prominent role as a vector of tick-borne diseases, but they can still act as reservoirs of bacteria and viruses (Sakamoto et al., 2016). A study of I. scapularis and Ixodes affinis microbiome by Van Treuren et al. (2015) in the East Coast and upper Midwest of United States found that male ticks had a wider variety of microbiome than females; similar results were observed in a study of I. scapularis in Texas by Thapa, Zhang, and Allen in 2019. In addition to being reservoir hosts, male ticks also play an important role in horizontal transmission of bacteria and viruses through processes such as co-feeding (Gonzalez et al., 1992; Labuda et al., 1993; Zemtsova et al., 2010; Belli et al., 2017). Here we show that male ticks carry just as much bacteria as female ticks and are therefore worthy of inclusion in future studies involving tick-borne diseases.

\subsection{Infection Prevalence of Bartonella spp. and B. Burgdorferi}

With regards to the two bacteria we tested, the data suggests a high prevalence of Bartonella spp. (75.16\%, Figure 2) and B. burgdorferi (47.13\%, Figure 2). The prevalence rate of B. burgdorferi will be discussed in the next section as it is synonymous with the coinfection rate of both bacteria. The results reported in this study is the highest reported prevalence rate for Bartonella spp. locally. A study by Carey et al. (2018) found $45.5 \%$ of I. scapularis ticks were infected with the bacteria compared to the $75.16 \%$ prevalence rate reported in this study. Another study by Curry et al. (2017) looked at female I. scapularis specifically and found 64\% prevalence rate of Bartonella spp. compared to the $76.83 \%$ reported prevalence rate of female ticks reported in this study. In both cases, there appear to be an increase in prevalence rate over time although more analysis is needed to test this hypothesis. The caveat here is that we used genus-level primers for Bartonella spp., which may factor into why we see a higher proportion of positive samples compared to the more specific $B$. burgdorferi primers. Further analysis using species-specific primers may provide a more accurate infection prevalence at the species level. However, for this study, we were interested in the concurrent infection of any form of Bartonella bacteria with B. burgdorferi and therefore the genus level primer was sufficient.

\subsection{Coinfection of Bartonella Spp. and B. Burgdorferi in Ticks}

Interestingly, all the ticks that had B. burgdorferi were also positive for Bartonella spp., but not all ticks that had Bartonella spp. were infected with $B$. burgdorferi. The coinfection prevalence was the number of positive $B$. burgdorferi samples of 74 (Table 2) out of the 157 total samples tested or $47.13 \%$ (Figure 1). This may imply that the presence of Bartonella spp. is essential for the infection of B. burgdorferi in the host tick, however additional studies will be required to address this claim.

The coinfection of Borrelia burgdorferi with other bacterial species and viruses has been documented previously in not only tick hosts (Holden et al., 2006; Sytykiewicz et al., 2012; Moutailler et al., 2016; Cross et al., 2018), but also organisms that ticks feed on such as Peromyscus leucopus (Hofmeister et al., 1998) and humans (Eskow, Rao and Mordechai, 2001). Studies have found that Borrelia burgdorferi is often found in ticks that are also infected with other bacterial species (Holden et al., 2006; Sytykiewicz et al., 2012; Moutailler et al., 2016; Cross et al., 2018), and to our 
knowledge, the $47.13 \%$ coinfection rate of B. burgdorferi and Bartonella spp. reported in this study is among the highest in the literature. The importance of the coinfection between these two bacteria extends beyond the host and even into a clinical setting in humans. A study by Eskow, Rao, and Mordechai in 2001 found that patients with neuroborreliosis conditions had DNA traces of Bartonella henselae and Borrelia burgdorferi, but no symptoms of cat-scratch disease.

\subsection{Implications of Study}

Surveillance of Lyme disease is becoming more prevalent but cat scratch disease is not being monitored consistently perhaps due to the asymptomatic nature in most infected individuals, something that is also a potential cause of under-diagnosed Lyme disease cases (Lloyd and Hawkins, 2018; Zangwill, 2021). Within Canada alone, cases of Lyme disease have increased from 144 cases in 2009 to 2025 cases in 2017 annually and is projected to continue increasing (Gasmi et al., 2017; Ogden et al., 2019). In contrast, cat scratch disease is not well documented in Canada whereas in the US, it is estimated to occur at a rate of 4-6 per 100,000 populations (Zangwill, 2021). Our high coinfection rates can be explained by the migration of ticks northward which has brought about various tick-borne diseases and increasing surveillance of these diseases and bacteria is warranted in order to better understand the spread and transmission of these diseases (Khatchikian et al., 2015; Sonenshine, 2018; Sagurova et al., 2019).

\section{Conclusion}

In this study, we determined the coinfection rate of Borrelia burgdorferi and Bartonella spp. that is consistent between sexes in Ixodes scapularis ticks. While both B. burgdorferi and Bartonella spp. are well studied individually, the relationship and interaction that these two bacteria have with each other and their host have not been thoroughly explored.

Here we outline several suggestions of research areas that may improve our understanding of the impact of coinfection in this system. The first is to look at the effects of bacterial load on the biology of the tick host. Focusing on the influence of the infection towards the metabolic pathways or behavior may contribute towards our understanding of parasite-host interaction and the mechanisms behind it. The findings that all the ticks that were positive for $B$. burgdorferi were also positive for Bartonella spp. but not the other way around is particularly fascinating. Further study on the molecular pathways of these bacteria inside the host and the role of coinfection in maintenance of B. burgdorferi should be considered. Finally, another aspect of this system that needs to be considered is the role that coinfection plays on the transmission of the bacteria. The high coinfection rate for both these bacteria may also imply a higher probability or bacterial load during transmission from the vector to a host and warrants further investigation. This is especially important from a clinical and public health point of view to manage the spread of Lyme disease and better understand the mechanism underlying the vector-host relationship.

To conclude, this study provides a foundation to further investigate the effect of coinfection in a highly successful vector system and raises the importance of considering other parasites when examining parasite-host interactions.

\section{Acknowledgements}

We would like to thank Dalhousie University for the continuous support for this research. We would also like to acknowledge all the students of BIOL3322 Parasitology class for their help with sample collection and organization. All the authors contributed equally to the study.

\section{References}

Batool, M., Blazier, J. C., Rogovska, Y. V., Wang, J., Liu, S., Nebogatkin, I. V., \& Rogovskyy, A. S. (2021). Metagenomic analysis of individually analyzed ticks from Eastern Europe demonstrates regional and sex-dependent differences in the microbiota of Ixodes ricinus. Ticks and Tick-borne Diseases, 101768.

Belli, A., Sarr, A., Rais, O., Rego, R. O., \& Voordouw, M. J. (2017). Ticks infected via co-feeding transmission can transmit Lyme borreliosis to vertebrate hosts. Scientific Reports, 7(1), 1-13.

Carey, K., El Nabbout, A., Macnutt-Burrows, E., Rooney, M., Mitton, M., Kho, J., ... Rossolimo, T. (2019). Physiology and Behaviour of Ixodes Scapularis Infected with Bartonella spp. in Nova Scotia. International Journal of Biology, 11(1).

Chang, C. C., Chomel, B. B., Kasten, R. W., Romano, V., \& Tietze, N. (2001). Molecular Evidence of Bartonella spp. in Questing Adult Ixodes pacificus Ticks inCalifornia. Journal of Clinical Microbiology.

Cross, S. T., Kapuscinski, M. L., Perino, J., Maertens, B. L., Weger-Lucarelli, J., Ebel, G. D., \& Stenglein, M. D. (2018). Co-infection patterns in individual Ixodes scapularis ticks reveal associations between viral, eukaryotic and bacterial microorganisms. Viruses, 10(7), 388. 
Curry, E., El Nabbout, A., Kho, J., Entremont, K. D., Jacks, F., \& Rossolimo, T. (2017). Effect of Bartonella spp. on the Freezing Tolerance in Female Ixodes scapularis. International Journal of Biology, 9(3), 65.

Diuk-Wasser, M. A., Vannier, E., \& Krause, P. J. (2016). Coinfection by Ixodes tick-borne pathogens: ecological, epidemiological, and clinical consequences. Trends in parasitology, 32(1), 30-42.

Edwards, J. F., \& Rawlings, J. A. (2012). Lyme disease in an experimental cat model. Lyme Borreliosis, 260, 187.

Eisen, R. J., \& Eisen, L. (2018). The blacklegged tick, Ixodes scapularis: an increasing public health concern. Trends in parasitology, 34(4), 295-309.

El Nabbout, A., Taylor, B. J., Kho, J., Mitton, M., \& Rossolimo, T. (2018). The Correlation of Francisella tularensis on Size and Supercooling of Dermacentor variabilis. International Journal of Biology, 10(2), 15.

Eskow, E., Rao, R. V. S., \& Mordechai, E. (2001). Concurrent infection of the central nervous system by Borrelia burgdorferi and Bartonella henselae: evidence for a novel tick-borne disease complex. Archives of neurology, 58(9), 1357-1363.

Foley-Eby, A. H., Savidge, C., \& Lloyd, V. K. (2020). Ixodes scapularis ticks and Borrelia burgdorferi on Prince Edward Island: Passive tick surveillance and canine seroprevalence. The Canadian Veterinary Journal, 61(10), 1107.

Gasmi, S., Ogden, N. H., Lindsay, L. R., Burns, S., Fleming, S., Badcock, J., .. Koffi, J. K. (2017). Surveillance for Lyme disease in Canada: 2009-2015. Canada communicable disease report $=$ Releve des maladies transmissibles au Canada, 43(10), 194-199. https://doi.org/10.14745/ccdr.v43i10a01

Ginsberg, H. S., Albert, M., Acevedo, L., Dyer, M. C., Arsnoe, I. M., Tsao, J. I., Mather, T. N., \& LeBrun, R. A. (2017). Environmental factors affecting survival of immature Ixodes scapularis and implications for geographical distribution of Lyme disease: the climate/behavior hypothesis. Plos one, 12(1), e0168723. doi:10.1371/journal.pone.0168723

Gonzalez, J. P., Camicas, J. L., Cornet, J. P., Faye, O., \& Wilson, M. L. (1992). Sexual and transovarian transmission of Crimean-Congo haemorrhagic fever virus in Hyalomma truncatum ticks. Research in virology, 143, 23-28.

Hofmeister, E. K., Kolbert, C. P., Abdulkarim, A. S., Magera, M. H., Hopkins, M. K., Uhl, J. R., .. Persing, D. H. (1998). Cosegregation of a novel Bartonella species with Borrelia burgdorferi and Babesia microti in Peromyscus leucopus. The Journal of Infectious Diseases.

Holden, K., Boothby, J. T., Kasten, R. W., \& Chomel, B. B. (2006). Co-detection of Bartonella henselae, Borrelia burgdorferi, and Anaplasma phagocytophilum in Ixodes pacificus ticks from California, USA. Vector-Borne \& Zoonotic Diseases, 6(1), 99-102.

Khatchikian, C. E., Prusinski, M. A., Stone, M., Backenson, P. B., Wang, I. N., Foley, E., ... Brisson, D. (2015). Recent and rapid population growth and range expansion of the Lyme disease tick vector, Ixodes scapularis, in North America. Evolution, 69(7), 1678-1689.

Kim, T. K., Tirloni, L., Pinto, A. F., Moresco, J., Yates III, J. R., da Silva Vaz Jr, I., \& Mulenga, A. (2016). Ixodes scapularis tick saliva proteins sequentially secreted every $24 \mathrm{~h}$ during blood feeding. PLoS neglected tropical diseases, 10(1), e0004323.

Labuda, M., Jones, L. D., Williams, T., Danielova, V., \& Nuttall, P. A. (1993). Efficient transmission of tick-borne encephalitis virus between cofeeding ticks. Journal of medical entomology, 30(1), 295-299.

Levin, M. L., \& Fish, D. (2000). Acquisition of coinfection and simultaneous transmission of Borrelia burgdorferi and Ehrlichia phagocytophila by Ixodes scapularis ticks. Infection and Immunity, 68(4), 2183.

Lloyd, V. K., \& Hawkins, R. G. (2018, December). Under-detection of Lyme disease in Canada. In Healthcare (Vol. 6, No. 4, p. 125). Multidisciplinary Digital Publishing Institute.

Massung, R. F., Slater, K., Owens, J. H., Nicholson, W. L., Mather, T. N., Solberg, V. B., \& Olson, J. G. (1998). Nested PCR assay for detection of granulocytic ehrlichiae. Journal of clinical microbiology, 36(4), 1090-1095.

Meeüs, T. D., Béati, L., Delaye, C., Aeschlimann, A., \& Renaud, F. (2002). Sex-biased genetic structure in the vector of Lyme disease, Ixodes ricinus. Evolution, 56(9), 1802-1807.

Müller, A., Reiter, M., Schötta, A. M., Stockinger, H., \& Stanek, G. (2016). Detection of Bartonella spp. in Ixodes ricinus ticks and Bartonella seroprevalence in human populations. Ticks and tick-borne diseases, 7(5), 763-767.

Moutailler, S., Moro, C. V., Vaumourin, E., Michelet, L., Tran, F. H., Devillers, E., ... Vayssier-Taussat, M. (2016). Co-infection of ticks: the rule rather than the exception. PLoS neglected tropical diseases, 10(3). 
Ogden, N. H., Trudel, L., Artsob, H., Barker, I. K., Beauchamp, G., Charron, D. F., ... Lindsay, L. R. (2006). Ixodes scapularis ticks collected by passive surveillance in Canada: analysis of geographic distribution and infection with Lyme borreliosis agent Borrelia burgdorferi. Journal of medical entomology, 43(3), 600-609.

Ogden, N. H., Bouchard, C., Badcock, J., Drebot, M. A., Elias, S. P., Hatchette, T. F., ... Webster, D. (2019). What is the real number of Lyme disease cases in Canada?. BMC Public Health, 19(1), 1-12.

Parola, P., \& Raoult, D. (2001). Ticks and tickborne bacterial diseases in humans: an emerging infectious threat. Clinical infectious diseases, 32(6), 897-928.

Patterson, J. W., Duncan, A. M., McIntyre, K. C., \& Lloyd, V. K. (2017). Evidence for genetic hybridization between Ixodes scapularis and Ixodes cookei. Canadian Journal of Zoology, 95(8), 527-537.

Ruef, C. (2011). Borrelia burgdorferi bacteremia, Infection.

Sagurova, I., Ludwig, A., Ogden, N. H., Pelcat, Y., Dueymes, G., \& Gachon, P. (2019). Predicted northward expansion of the geographic range of the tick vector Amblyomma americanum in North America under future climate conditions. Environmental health perspectives, 127(10), 107014.

Sakamoto, J. M., Ng, T. F. F., Suzuki, Y., Tsujimoto, H., Deng, X., Delwart, E., \& Rasgon, J. L. (2016). Bunyaviruses are common in male and female Ixodes scapularis ticks in central Pennsylvania. PeerJ, 4, e2324.

Sonenshine, D. E. (1991). Biology of ticks. Vol 1. New York: Oxford UP.

Sonenshine, D. E. (2018). Range expansion of tick disease vectors in North America: implications for spread of tick-borne disease. International Journal of Environmental Research and Public Health, 15(3), 478.

Steiner, F. E., Pinger, R. R., Vann, C. N., Grindle, N., Civitello, D., Clay, K., \& Fuqua, C. (2014). Infection and co-infection rates of Anaplasma phagocytophilum variants, Babesia spp., Borrelia burgdorferi, and the rickettsial endosymbiont in Ixodes scapularis (Acari: Ixodidae) from sites in Indiana, Maine, Pennsylvania, and Wisconsin. Journal of Medical Entomology, 45(2), 289-297.

Sytykiewicz, H., Karbowiak, G., Werszko, J., Czerniewicz, P., Sprawka, I., \& Mitrus, J. (2012). Molecular screening for Bartonella henselae and Borrelia burgdorferi sensu lato co-existence within Ixodes ricinus populations in central and eastern parts of Poland. Annals of Agricultural and Environmental Medicine, 19(3).

Thapa, S., Zhang, Y., \& Allen, M. S. (2019). Bacterial microbiomes of Ixodes scapularis ticks collected from Massachusetts and Texas, USA. BMC microbiology, 19(1), 1-12.

Van Treuren, W., Ponnusamy, L., Brinkerhoff, R. J., Gonzalez, A., Parobek, C. M., Juliano, J. J., Andreadis, T. G., Falco, R. C., Ziegler, L. B., Hathaway, N., Keeler, C., Emch, M., Bailey, J. A., Roe, R. M., Apperson, C. S., Knight, R., \& Meshnick, S. R. (2015). Variation in the microbiota of Ixodes ticks with regard to geography, species, and sex. Applied and environmental microbiology, 81(18), 6200-6209.

Wills, M. K., Kirby, A. M., \& Lloyd, V. K. (2018). Detecting the lyme disease spirochete, Borrelia burgdorferi, in ticks using nested PCR. JoVE (Journal of Visualized Experiments), (132), e56471.

Wójcik-Fatla, A., Cisak, E., Chmielewska-Badora, J., Zwolinski, J., Buczek, A., \& Dutkiewicz, J. (2006). Prevalence of Babesia microti in Ixodes ricinus ticks from Lublin region (eastern Poland). Annals of Agricultural and Environmental Medicine, 13(2), 319.

Zangwill, K. M. (2021). Cat Scratch Disease and Bartonellaceae: The Known, the Unknown and the Curious. The Pediatric Infectious Disease Journal, 40(5S), S11-S15.

Zemtsova, G., Killmaster, L. F., Mumcuoglu, K. Y., \& Levin, M. L. (2010). Co-feeding as a route for transmission of Rickettsia conorii israelensis between Rhipicephalus sanguineus ticks. Experimental and Applied Acarology, 52(4), 383-392.

\section{Copyrights}

Copyright for this article is retained by the author(s), with first publication rights granted to the journal.

This is an open-access article distributed under the terms and conditions of the Creative Commons Attribution license (http://creativecommons.org/licenses/by/4.0/). 